Objective To investigate cognitive function in obese Egyptian adolescents.

Methods A stratified-cluster proportionate random sampling was used to select a representative sample of 4899 pupils in preparatory school aged $12-15$ year. Overweight was defined as BMI $\geq 85$ th but $<95$ th percentile, while obesity as $\geq 95$ th BMI percentile, using Egyptian percentile. Wechsler Intelligence Scale and Executive Function test were used to assess cognitive function.

Results The prevalence of overweight was $14.9 \%$ and obesity was $6.6 \%$. The children's mean full scale IQ was 89.54 . Wechsler Intelligence Scale revealed significant differences in performance intelligence quotient (PIO) scores between overweight and obese children. Parental educational was significantly related to total intelligence quotient (TIO) (p 0.03). Executive function was significantly impaired in obese adolescents. Executive function was significantly lower in obese than overweight adolescents. After adjustment for age and parental education level female obese adolescents had lower cognitive function than male adolescents. BMI and waist circumference were the best predictor of impaired cognitive function in obese and overweight adolescents.

Conclusion Overweight and obesity were associated with cognitive dysfunction in adolescents.

\section{OBESITY - ADOLESCENT PROBLEM}

doi:10.1136/archdischild-2012-302724.1430

'B Stanimirov, ${ }^{2} \mathrm{M}$ Jankovic, ${ }^{3} \mathrm{M}$ Djordjevic. 'Pediatrics, Health Centre Novi Sad, Novi Sad; 'Pediatrics, Health Centre Cuprija, Cuprija; ${ }^{3}$ Paedaitrics, Health Centre Gornji Milanovac, Gornji Milanovac, Serbia

Background Obesity is one of the most serious health problems of the modern world.

Aim Was to assess the prevalence of obesity in adolescents, who are treated in the school infirmary "SMT" Health Center Novi Sad. Material and Methods The study was conducted among high school students across the measurement of anthropometric parameters and calculate body mass index. The students answered questions about the quality and quantity of food and liquid intake, frequency and duration of physical activity or no activity.

Results The study lasted over two academic years (2009 to 2011.), The number of učenika. Gojaznih $92.93 \%$ : $16.23 \%$, fed: $4.49 \%$ ideal body weight had: $72.21 \%$ of students. Rolls for meals consumed $62.47 \%$ of adolescents, the liquid entered in the form of soda $71.82 \%$, not soda and water was used by $21.12 \%$, adolescents consumed sweets daily: $80.85 \%$ of adolescents. Only $39.57 \%$, daily participation in the sport, and they rarely participate $9.23 \%, 73.56 \%$ of them even sit in front of the TV or computer, with 2 or more hours a day.

Conclusion The consequence of obesity is sedentary lifestyle, lack of exercise, improper and inadequate and Save. Preventive measures should focus on: diet, physical activity, reducing sedentary habits, which is a prerequisite for the prevention of possible consequences made in adulthood.

\section{ATHEROSCLEROSIS RISK FACTORS IN OBESE CHILDREN AND ADOLESCENTS}

doi:10.1136/archdischild-2012-302724.1431

S Atef, T Adham. Ain Shams University, Cairo, Egypt

Introduction Atherosclerosis is among the important long term complications and leading causes of death among obese children and adolescents. Its risk factors (RFs) include mainly: high body mass index (BMI), central obesity, smoking, lack of physical activity (PA), hypertension, hyperglycemia, elevated uric acid (UA), alanine aminotransferase (ALT), inflammation, adhesion molecules (as E-selectin) and highly sensitive (hs) CRP.
Aim of the work To determine the extent and severity of the aforementioned obesity-related atherosclerotic risk factors among school aged children and adolescents.

Subjects and methods The sample has included 98 obese (nonsyndromic) and 36 non obese control subjects aged 6-16 years. A questionnaire was filled to evaluate the daily and weekly PA calculated in hours, anthropometry was done and blood pressure was measured, together with assessment of serum lipid profile and levels of fasting blood sugar, ALT, UA, E-selectin and hs CRP.

Results $55 \%$ of obese group have shown 4 or 5 atherosclerotic RFs. One or more features of abnormal lipid profile were found in $94 \%$ of obese group with 73\% showing high cholesterol level. ALT and UA were significantly higher in the obese group, similarly E-selectin that was elevated in $71 \%$ of obese and hs CRP were significantly higher among obese. FBS did not show similar significant elevations. Positive correlations were found between cholesterol, E-selectin and hs CRP with BMI and waist/hip ratio.

Conclusion Most of obese children and adolescents do suffer from some risk factors that can lead to an earlier and greater risk for developing atherosclerosis.

\section{CHILDREN AND OBESITY - HOW SEVERE IS THE PROBLEM?}

doi:10.1136/archdischild-2012-302724.1432

${ }^{1}$ BE Popovici, ${ }^{2} \mathrm{M}$ Mitrica. 'Faculty of Medicine, 'Transilvania' University Brasov; 2Pediatrics, Faculty of Medicine 'Transilvania' University Brasov, Brasov, Romania

Introduction Childhood is more ofen nowadays confronting with a different pathology, specific to adulthood like obesity, diabetes mellitus, essential hypertension and hypercholesterolemia. The prevalence of these diseases is rising and cardiovascular risk factors are present even in children and the key is represented by the process of atherosclerosis.

Objective The aim of our study is to determine the presence of cardiovascular risk factors in childhood and if there is any evidence of atherosclerosis effects on the vessels.

Material and method The study had included 80 children, boys and girls, aged between 10 and 18 years. All of the children had obesity. The protocol of investigations had consisted in: blood pressure measurement (BP), body mass index calculation (BMI), blood levels of glucose, oral test of glucose tolerance, cholesterol, tryglycerides, low density lipoproteins, high density lipoproteins, echocardiography and measurement of the mass of the left ventricle, Doppler ecograpy of the common carotid artery and measurement of the intima-media thickness (IMTc) as a evidence of the atherosclerotic process.

Results We had found a stong and positive correlation between IMTc and BMI, systolic BP, the level of total cholesterol, tryglycerides and a negative correlation with the level of high density lipoproteins. All the subjects had had impairment of the oral test of glucose tolerance.

Conclusions The rising incidence of obesity in children is a reality. It is strongly connected with the atherosclerosis and its consequences, like early structural changes of artery, even in childhood, and therefore prevention should be a priority.

\section{VITAMIN D DEFICIENCY AND SECONDARY HYPERPARATHYROIDISM IN OBESE CHILDREN}

doi:10.1136/archdischild-2012-302724.1433

'R Ghergherehchi, ${ }^{2 N}$ Hazhir, ${ }^{2} \mathrm{~A}$ Tabrizi. 'Pediatric Endocrinology, Tabriz University of Medical Sciences; ${ }^{2}$ Tabriz Children Hospital University of Medical Sciences, Tabriz, Iran

Obesity subjects individuals into metabolic and endocrine disorders. Thus obesity may increase the risk of vitamin $\mathrm{D}$ deficiency. This text aims at studying the prevalence of vitamin D deficiency and secondary hyperparathyroidism in obese children. In a non-randomized 